\title{
Layout of the MICE Demonstration of Muon Ionization Cooling
}

\section{Colin Whyte ${ }^{\dagger}, a$ Jaroslaw Pasternak, $b$ and Chris Hunt, $b$}

a University of Strathclyde

b Imperial College London

E-mail: colin.whyte@strath.ac.uk, j.pasternak@imperial.ac.uk,

christopher.hunt08@imperial.ac.uk

\section{for the MICE collaboration}

\begin{abstract}
Muon beams of low emittance provide the basis for the intense, well-characterised neutrino beams necessary to elucidate the physics of flavour at the Neutrino Factory and to provide multi-TeV lepton-antilepton collisions at the Muon Collider. The international Muon Ionization Cooling Experiment (MICE) will demonstrate muon ionization cooling, the technique proposed to reduce the phase-space volume occupied by the muon beam at such facilities. In an ionization-cooling channel, the muon beam traverses a material (the absorber) losing energy, which is replaced using RF cavities. The combined effect is to reduce the transverse emittance of the beam (transverse cooling). The configuration of MICE required to deliver the demonstration of ionization cooling was prepared in parallel to the execution of a programme designed to measure the cooling properties of liquid-hydrogen and lithium hydride (Step IV). The design will be presented together with a summary of the projected performance of the experiment.
\end{abstract}

The European Physical Society Conference on High Energy Physics

5-12 July

Venice, Italy

\footnotetext{
${ }^{*}$ Work supported by the (UK) Science and Technology Facilities Council and the (US) Dept. of Energy and National Science Foundation

${ }^{\dagger}$ Speaker.
} 


\section{Introduction}

Stored muon beams have been proposed as the source of neutrinos at the Neutrino Factory and as the means to deliver multi-TeV lepton-antilepton collisions at the Muon Collider [1]. The tertiary muon beam occupies a large volume in phase space. To optimise the intensity, while maintaining a small aperture in the acceleration systems, requires that the phase space is reduced. Traditional cooling techniques are inefficient for muons. Ionization cooling, in which the beam is passed through material (an absorber) and subsequently accelerated, is the technique by which it is proposed to cool the beam $[2,3]$. A factor of $10^{5}$ in $6 \mathrm{D}$ muon cooling has been achieved in simulation [4], however this technique has never been demonstrated experimentally.

A full demonstration of transverse ionization cooling is the goal of the MICE collaboration. The current form of the experiment, MICE Step IV, is providing the data required to study the material properties of typical absorber materials, lithium hydride $(\mathrm{LiH})$ and liquid hydrogen, and the behaviour of muon beams in complex solenoidal fields [5]. The addition of an accleration stage however, is essential for the demonstration of ionization cooling.

Cooling performance depends on the initial emittance and momentum of the beam, the absorber material and the transverse betatron function $\left(\beta_{\perp}\right)$ at the absorber. Ionization cooling is optimised when $\beta_{\perp}$ is at a minimum in the absorber and initial emittance is large. Additionally, the beam momentum must be selected to optimise ionization energy losses, whilst the material must be chosen to reduce the emittance growth due to the effect of scattering.

\section{Design and Development}

\subsection{Physical Layout}

The configuration that will be used for the demonstration of ionization cooling is shown in Fig. 1. It contains two single $201 \mathrm{MHz}$ cavities, one primary $(65 \mathrm{~mm}) \mathrm{LiH}$ absorber, and two secondary $(32.5 \mathrm{~mm}) \mathrm{LiH}$ "screening" absorbers. The central absorber is sandwiched between two superconducting "focus-coil" (FC) modules. The beam enters and exits the cooling cell through two spectrometer solenoid (SS) modules

The emittance is measured upstream and downstream of the cooling channel using the Scintillating Fibre trackers, placed within a uniform $4 \mathrm{~T}$ solenoid field [6]. Instrumentation upstream and downstream of the cooling channel provides PID and event selection information [7].

\subsection{Magnetic Design}

The lattice has been designed to maximise the measurable reduction in transverse emittance, by matching the betatron function to a minimum at the central absorber, whilst moderating the maximum value within the FC modules. This reduces the influence of non-linear effects and permits secondary absorbers to be installed close to the secondary minima enhancing the cooling effect and shielding the trackers from RF-induced noise. The phase advance of the cooling cell was carefully chosen to minimize the chromatic effect due to the large momentum spread of the beam, which leads to a chromatic mismatch at the primary absorber and in the downstream spectrometer.

The resulting on axis magnetic field strength is shown in Fig. 2 (left) for the three planned settings $(140,200$ and $240 \mathrm{MeV} / \mathrm{c})$. The downstream FC and SS modules are powered in the 


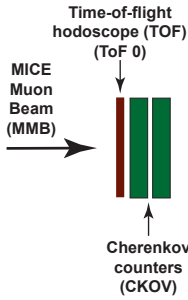

MICE

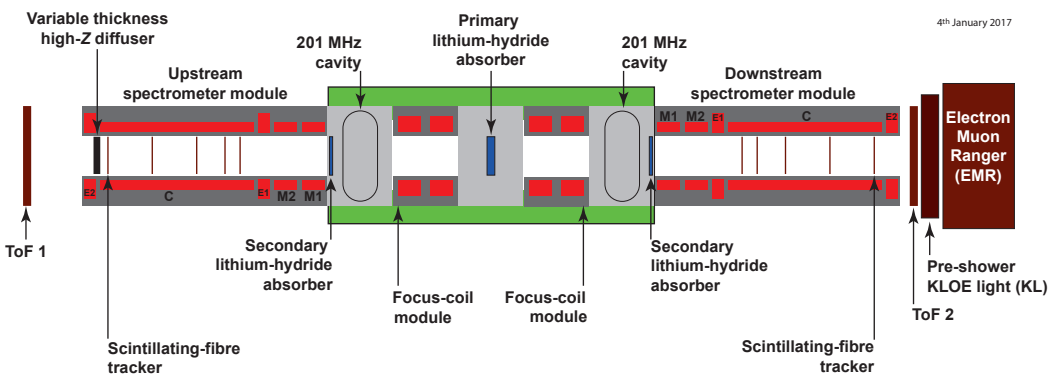

Figure 1: Layout of the lattice configuration for the cooling demonstration. The red rectangles represent the solenoids. The individual coils are labelled E1, C, E2, M1 and M2. The ovals represent the RF cavities and the blue rectangles the absorbers. The various detectors (time-of-flight hodoscopes, Cerenkov counters, scintillating-fibre trackers, KLOE Light calorimeter, electron muon ranger) used to characterise the beam are also represented. The green-shaded box indicates the cooling cell.

opposite sense to the upstream FC and SS modules so that the field changes sign at the primary absorber. This is a desirable feature for studying the cancellation of canonical angular momentum through the lattice.
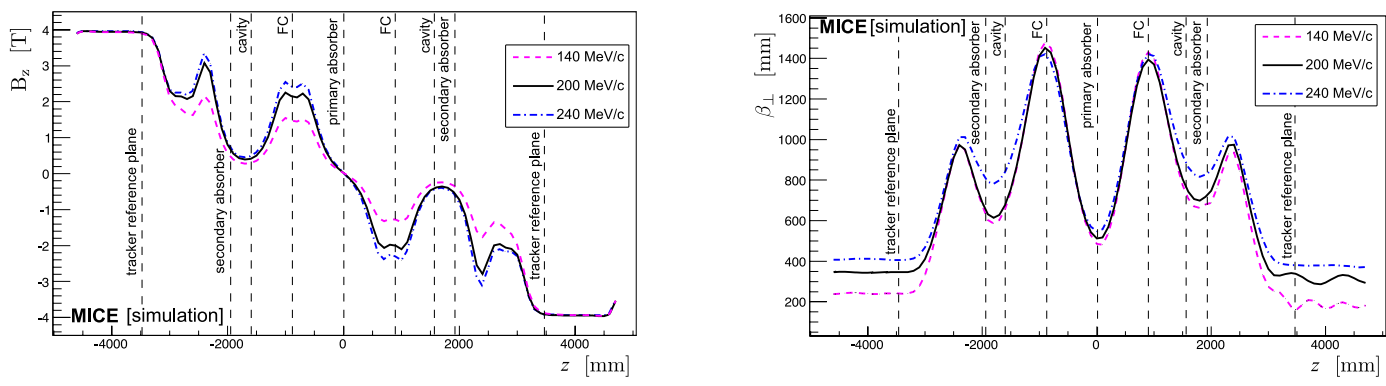

Figure 2: On the left, longitudinal magnetic field strength through the length of the cooling channel for the same settings. On the right, $\beta_{\perp}$ through the length of the cooling channel for the 200 (solid black line), 140 (dashed purple line) and $240 \mathrm{MeV} / \mathrm{c}$ (mixed blue line) settings. The position of key components are indicated by the vertical lines.

\subsection{Optical Layout}

The betatron functions shown in Fig. 2 (right) are matched for different initial momentum beams. In all cases, the Courant Snyder parameter $\alpha$ was matched to zero within the tracking volume, and a minimum value of beta in the central absorber was achieved. The matching procedure takes into account the change in momentum of the beam.

\section{Performance}

The performance of the lattice has been evaluated using the official simulation and reconstruction software of the MICE Collaboration: the MICE Analysis and User Software (MAUS [8]). Each of the 3 momenta beams were initially simulated as matched within the upstream spectrometer and 
allowed to propagate through to the EMR detector. A range of emittances from 2 to $12 \mathrm{~mm}$ were selected to probe the measurement space available to the lattice. Fig. 3 (left) shows the emittance behaviour for a $200 \mathrm{MeV} / \mathrm{c}$ muon beam, simulated with an initial emittance of $6 \mathrm{~mm}$.
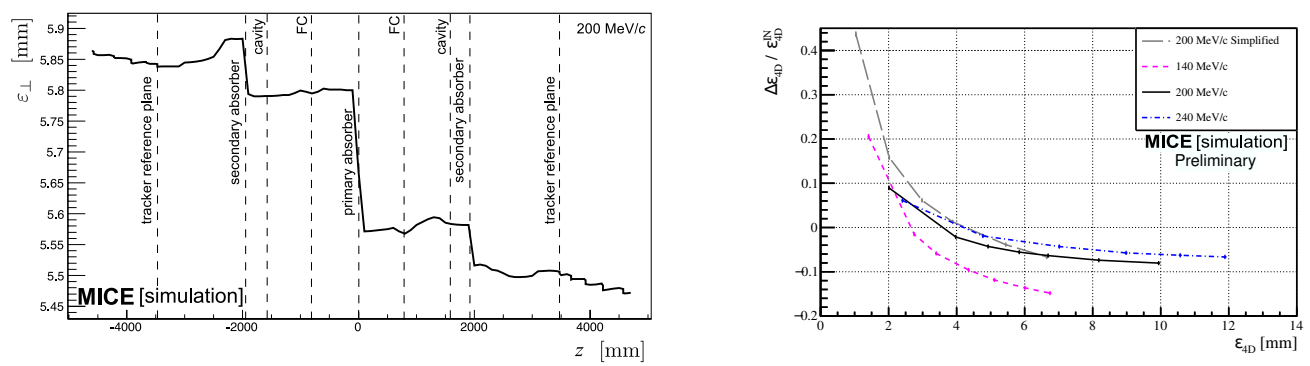

Figure 3: On the left, evolution of the transverse emittance for a transmitted $6 \mathrm{~mm}$ beam, with central momentum $200 \mathrm{MeV} / \mathrm{c}$ (RMS spread 4.0\%). On the right, the fractional change in transverse emittance for each transmitted beam. The $200 \mathrm{MeV} / \mathrm{c}$ setting for the simplified design is also included for comparison.

The effects of the absorbers are clearly recognisable as distinct drops in the emittance. In general, good behaviour of the emittance is maintained through the solenoidal fields, with some small non-linear effects, due to the high focussing strength of the SS match coils.

Fig. 3 (right) shows the fractional change in emittance between the upstream and downstream tracker reference planes, for particles that were transmitted successfully. A statistically significant effect of transverse cooling is measurable for all three momenta, and across a range of initial emittances. The transmission of each beam is over $95 \%$ for all but the largest initial emittances.

\section{Simplified Design}

In order to mitigate issues arising from the damaged M1 coil of the downstream SS module, a simplified layout has also been considered. The design removes the downstream spectrometer module leaving a drift space following the second RF cavity.

Fig. 4 (left) depicts the layout of the downstream section of the cooling cell. The SS module has been removed and replaced with a bespoke 3-station tracking detector in the zero-field region. The downstream emittance is calculable using a combination of a straight line track fit and a total momentum reconstruction using the EMR detector. There are several benefits of this design, predominantly the low cost. No repairs are required to the downstream SS; the cooling channel fits entirely within the existing magnetic return yoke; and the only new piece of equipment is the 3 -station tracker module, for which all the components are already in hand.

Due to the lack of focussing into the downstream detectors, the optics becomes limited by the aperture of the 3-station tracker. Fig. 4 (right) demonstrates the growth of the betatron function due the drift space. Only a subset of emittances can therefore be successfully transmitted, however cooling can still be measured with high precision for a range of momenta and emittances. In contrast to the original design however, the transmission is reduced for initial emittances, $\varepsilon>5 \mathrm{~mm}$. 

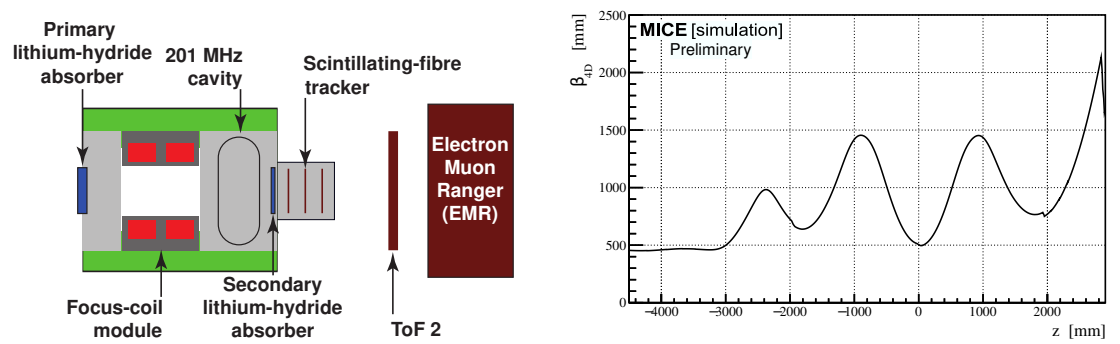

Figure 4: On the left, layout of the downstream components of the simplified design. The spectrometer solenoid has been replaced by a 3-station tracker. On the right, evolution of the transverse betatron function for the $200 \mathrm{MeV} / \mathrm{c}$ setting in this lattice. Significant growth can be seen, following the secondary absorber at $z=2000 \mathrm{~mm}$, due to the lack of magnetic fields.

\section{Conclusion}

The MICE collaboration has presented a design that is capable of demonstrating ionization cooling and measuring the effect to a high precision, across a range of parameters. The equipment is either in hand, or at an advanced stage of preparation, and the experimental performance has been studied in detail. An additional design for a simplified configuration has also been considered as a way to mitigate risk and cost if required.

\section{References}

[1] Geer, S, Neutrino beams from muon storage rings: Characteristics and physics potential, Phys. Rev. D 57, no.11, p. 6989, 1998.

[2] A.N.Skrinsky et.al., Cooling methods for beams of charged particles, Sov. J. Part. Nucl. 12, no. 223, 1981.

[3] D. Neuffer, Principles and Applications of Muon Cooling, Part. Accel., 14, pp. 75âĂŞ90, 1983.

[4] D. Stratakis and R. B. Palmer, Rectilinear six-dimensional ionization cooling channel for a muon collider: A theoretical and numerical study, Phys. Rev. ST Accel. Beams, 18, p. 031003, Mar 2015.

[5] P. Franchini et al., Results from MICE Step IV, IPAC Conf. Proc., 2017. WEPAB130.

[6] A. Dobbs et al., The reconstruction software for the mice scintillating fibre trackers, Journal of Instrumentation, 11, no. 12, p. T12001, 2016.

[7] D.Orestano, The detector system of the MICE experiment, Nucl. Inst. Phys. A, vol. 617, pp. 45 âĂŞ 47, 2010.

[8] C. D. Tunnell and C. T. Rogers, MAUS: Mice User Analysis Software, IPAC Conf. Proc., 2011. IPAC-2011-MOPZ013. 